\title{
Hacia una narrativa de la naturaleza: la psicología ante el reto sustentable*
}

\author{
Cristóbal Bravo F. ${ }^{* *}$
}

\begin{abstract}
Resumen: El modelo de desarrollo sustentable ha motivado la generación de una importante producción científica en distintos ámbitos. Tal ha sido el caso de la psicología que se ha propuesto comprender el papel de los factores conductuales y mentales implicados en la relación del hombre con la naturaleza. Si bien el emergente campo de la psicología ambiental se ha planteado objetivos pertinentes para el fomento de conductas sustentables, ésta ha operado desde modelos epistemológicos que tienden a mantener elementos de la cosmovisión moderna relacionada con el deterioro ambiental. Se proponen las aproximaciones narrativas de la psicología como una alternativa capaz de subvertir el orden epistemológico.

Palabras clave: psicología, desarrollo sustentable, medioambiente, narrativas del sí mismo
\end{abstract}

\section{Towards a narrative of nature: psychology in the face of sustainable challenge}

\begin{abstract}
Considerable scientific research in different fields has been motivated by sustainable development model. Such is the case of psychology which looks for behavioral and mental factors that take place in human-nature relations. Even though the emerging field of environmental psychology has proposed itself stimulating sustainable conducts, it has been working within epistemological frameworks that tend to maintain modern worldview approaches related to environmental damage. Narrative approaches of psychology are suggested here as an alternative method capable of subverting the epistemological order.

Keywords: psychology, sustainable development, nature environment, narratives of the self
\end{abstract}

\section{Rumo a uma narrativa da natureza: a psicologia diante do desenvolvimento sustentável}

Resumo: O modelo de desenvolvimento sustentável tem motivado a geração de uma importante produção científica em diferentes âmbitos. Tal foi o caso da psicologia, que tem proposto compreender o papel dos fatores comportamentais e mentais envolvidos na relação do homem com a natureza. Enquanto o campo emergente da psicologia ambiental almeja objetivos pertinentes para o fomento de condutas sustentáveis, que tem operado com base em modelos epistemológicos

\footnotetext{
* Este trabajo está inserto en el marco del proyecto de investigación FONDECYT 1090455 "Los Paisajes del Agua: prácticas sociales y sustentabilidad en la cuenca hidrográfica del río Valdivia” y es parte de los avances de la tesis doctoral del autor.

** Universidad Austral de Chile, Valdivia, Chile. Email: bravocristobal@hotmail.com
} 
que tendem a manter elementos da cosmovisão moderna relacionada à deterioração ambiental. São propostas aproximações narrativas da psicologia como uma alternativa capaz de subverter a ordem epistemológica.

Palavras-chave: psicologia, desenvolvimento sustentável, meio ambiente, auto-narrativas

Recibido: 22.11.2009

Aceptado: 02.05.2010

\section{Introducción}

Alcanzar los objetivos de un modelo de desarrollo sustentable exige un replanteamiento profundo de la relación del hombre con la naturaleza, asunto que se ha convertido progresivamente en un desafío teórico y práctico para las ciencias sociales en los últimos veinte años. La psicología se ha visto fuertemente interpelada por los imperativos del paradigma sustentable, generándose al respecto una importante producción científica agrupada bajo el rótulo de psicología ambiental. Estos desarrollos han logrado demostrar la importancia del ámbito conductual en los problemas ambientales por medio de la identificación de comportamientos y la comprensión e intervención en variables psicológicas implicadas en el cuidado del medioambiente. El énfasis de estos trabajos ha estado puesto en el comportamiento individual, asumiendo que los conocimientos tienen un carácter universal, independiente del contexto en el que se producen. Tal enfoque ha conducido a considerar al sujeto como un agente pasivo en la producción de conocimientos acerca del medioambiente, y consecuentemente, a desestimar las versiones que tienen los propios sujetos (Weisenfeld, 2003).

Ubicar los problemas medioambientales y la relación del hombre con la naturaleza dentro de las preocupaciones de la psicología requiere adoptar una mirada distinta a la que ha dominado tradicionalmente a la psicología experimental. Las orientaciones actuales en psicología social han insistido en mostrar que la realidad social no es independiente de lo que podamos pensar de ella (Ibáñez, 1994). Esta misma fórmula puede aplicarse para el caso de la sustentabilidad y podemos plantear: lo que es “en realidad” la naturaleza depende en gran medida de cómo la representamos, es decir, qué pensamos de ella y cómo la imaginamos.

El comportamiento social está fuertemente arraigado en los sentidos que las personas construyen acerca de sus propias acciones, de los objetos y de los otros individuos. Entendida de este modo la realidad social asume un carácter simbólico y dinámico ya que las personas en sus interacciones cotidianas y procesos comunicativos se orientan a la negociación o búsqueda de acuerdo sobre los significados subjetivos. Una orientación cualitativa en psicología que busque "descubrir la naturaleza del mundo social a través de la comprensión de cómo la gente actúa y da senti- 
do a sus propias realizaciones vitales" (Alonso, L. E., 1998: 27) constituye una fértil aproximación a la subjetividad social, cuestión que la psicología ambiental hasta ahora ha relegado de manera evidente.

Si las ciencias sociales están llamadas a ejercitar su compromiso ético con el medioambiente, estas deben hacerlo desde un marco epistemológico que promueva acciones humanas capaces de recomponer o integrar una visión fragmentada de la realidad, de la que son en gran medida responsables las prácticas e instituciones modernas. Darle una oportunidad al sujeto, lograr que pueda contar su propia historia y desplegar su visión de las cosas sin constreñimientos objetivos, constituye en este sentido un desafío teórico y metodológico para aquellas ciencias abocadas al comportamiento social.

\section{El desafío sustentable implica modificar la relación hombre-naturaleza}

Probablemente el concepto de Desarrollo Sustentable ha sido una de las propuestas más consistentes y de mayor reconocimiento global ante la crisis ambiental que enfrentamos. A más de treinta años de que comienza a emplearse, el término Desarrollo Sustentable ha estado presente en la agenda política nacional e internacional y se ha incorporando gradualmente a los distintos discursos sociales, llegando a transformarse hoy en día en un sinónimo de lo políticamente correcto. En este sentido se destaca cómo el concepto ha sido capaz de penetrar en distintos ámbitos del conocimiento, suscitando discusiones de marcado carácter interdisciplinar.

Si bien existen distintas acepciones del término, estas no difieren en lo sustancial. Como lo sugiere su definición mayormente aceptada, el Desarrollo Sustentable es aquel "que satisface las necesidades básicas y las aspiraciones de bienestar de la población presente, sin comprometer la capacidad de las futuras generaciones para la satisfacción de sus necesidades y aspiraciones” (Brundtland, 1987: 22). Con tales objetivos, la Cumbre de Río del año 1992, conocida como la Cumbre de la tierra, generó una serie de acuerdos internacionales que apuntaban a enfrentar la situación ambiental del planeta, sobretodo el fenómeno del cambio climático, con un modelo que ratificaba la necesidad de promover un desarrollo basado en el equilibrio de las dimensiones económicas, sociales y ambientales.

Probablemente uno de los mayores méritos del modelo de desarrollo sustentable ha sido su contribución a la creación de una suerte de lenguaje común acerca del impacto del hombre en el medioambiente. Este conjunto de conceptos posibilita la comunicación de fenómenos producidos en distintos contextos al identificar los principales problemas que afectan hoy al medioambiente, sus causas y sugerir ciertas vías por donde debieran transitar las sociedades hacia el desarrollo. A pesar de esta contribución, existen ciertas críticas que apuntan al carácter antropocéntrico del 
modelo, así como a ciertas ambigüedades respecto a las necesidades a las que se refiere, de sus límites y de la forma cómo anticipar las necesidades cambiantes (Munasingue \& McNeely, 1995, en Weisenfeld, 2003).

Si bien el paradigma de la sustentabilidad logra reconocer que tras estas problemáticas existe una causalidad compleja que involucra factores económicos, sociales y ambientales, incrementadas dramáticamente en el último siglo, es evidente que las raíces del deterioro ambiental acompañan al hombre desde mucho antes. Existen suficientes antecedentes históricos, sociológicos y antropológicos como para plantear que la cosmovisión occidental tiende a considerar al hombre y a la naturaleza como entidades separadas, donde el hombre se concibe a sí mismo como superior por sobre el resto de las cosas, orientando sus acciones de acuerdo a una racionalidad instrumental.

En un conocido artículo publicado en la revista Science en 1967, el historiador Lynn White exponía una serie de antecedentes históricos, como el uso de tecnologías, creencias y suposiciones vinculadas a una cosmovisión moderna que han tenido desenlaces nefastos para el medioambiente. Según White, la tradición judeo-cristiana, principalmente a través de las sagradas escrituras, sería en gran parte responsable de nuestra crisis ambiental, ya que al conceptualizar al hombre como un ser superior con respecto a las otras criaturas vivientes, posibilita una interpretación antropocéntrica que conduce al despliegue de tecnologías destinadas a dominar la naturaleza. White, al igual que para un conjunto de científicos, que durante la década de 1970 desarrollaron una vertiente de pensamiento conocida como "la ética ambiental”, desecha las soluciones científicas y técnicas, proponiendo que para enfrentar la crisis es imprescindible una reformulación del sentido de la relación hombre-naturaleza.

La cosmovisión occidental está constituida sobre la base de un pensamiento dualista que desde Descartes en adelante ha sido considerado como el mejor vehículo para alcanzar el Progreso. Separaciones ontológicas como mente-cuerpo, sujeto-objeto y hombre-naturaleza han conformado, de este modo, el esquema con el que esta civilización ha interpretado los fenómenos que le inquietan y con el que ha concebido las soluciones a los problemas que se le van presentando. Estas ideas se han extendido hacia los distintos ámbitos de la vida social y se han incorporado de tal modo a nuestra vida cotidiana que han tendido a ser consideradas como parte de la naturaleza humana.

Sería injusto considerar que esta forma de pensamiento que comporta las raíces de la crisis ambiental es extensiva a todas las culturas que habitan el planeta. Es necesario circunscribir esta cosmovisión a la Modernidad occidental, ya que tal como lo demuestra una serie de estudios antropológicos existen culturas originarias cuya relación con naturaleza está basada en un profundo sentido de interdependencia donde seres humanos y no humanos son considerados como similares (ver por ejemplo, Descola \& Palsson, 2001). 
Los patrones de pensamiento implicados en una trayectoria dañina para el medioambiente se encuentran profundamente arraigados en las instituciones modernas; en la economía de mercado, en la ciencia, en la educación, en las leyes, etc. Estas pautas de pensamiento atraviesan en las dimensiones social, cultural y psicológica, del mundo occidental. No resulta fácil despojarse de hábitos formados a lo largo de siglos y de los cuales nuestras sociedades se han vuelto dependientes.

El desafío de la sustentabilidad supone un cambio en los modos de pensar, sentir y actuar de las personas, así como una modificación de la sociedad a gran escala. Ruckelshaus (1989) sostiene que para lograr un cambio en la trayectoria insostenible se requiere de una transformación de magnitud comparable a lo que ha sido la Revolución Agrícola a fines de la era Neolítica y a la Revolución Industrial de los últimos dos siglos. Desde esta perspectiva se asume que para enfrentar los principales problemas ambientales más serios del planeta tales como el calentamiento global, la pérdida de la biodiversidad, la contaminación de las aguas, la desforestación y el agotamiento de las tierras para la agricultura, entre otros, se hace necesaria una modificación en las dimensiones social, cultural y psicológica del ser humano. En este sentido las ciencias sociales se ubican como una de las principales fuentes para la comprensión las formas de pensamiento implicadas en el deterioro del medioambiente así como en la modificación de estas.

\section{La aproximación de la psicología ambiental}

La psicología ambiental en sus inicios estuvo "fuertemente influenciada por las cuestiones que destacan los arquitectos, los urbanistas u otros profesionales del acondicionamiento del cuadro urbano”, siendo entonces de gran utilidad la teoría de campo de Kurt Lewin que apuntaba a que la conducta es una función de una situación que comprende las condiciones del individuo y las variables del ambiente (Moser, 2003). Sin embargo será el modelo de desarrollo sustentable el que tendrá una importancia determinante en el futuro de la disciplina, y que hará que esta visualice una potencial contribución a la generación de cambios sociales en materia de protección del medioambiente (Velg \& Steg, 2007). La identificación de los principales problemas del medioambiente y el reconocimiento de sus causas por parte de una serie de organismos multilaterales motivará la confianza de los psicólogos en el logro de resultados positivos. En tanto el estado del medioambiente se considera en cierta medida dependiente de un conjunto de conocimientos, creencias y actitudes, esta trayectoria sería modificable (Howard, 2000).

En la última década se ha suscitado una proliferación de publicaciones, sobretodo en Europa. Estas investigaciones han dado lugar a diversos libros, Handbooks y revistas especializadas. Tal como sostiene Weisenfeld (2003) en la producción científica se advierte una evidente postergación de la dimensión social de las conductas implicadas en el cuidado del 
medioambiente, así como también de las variables subjetivas y de los procesos de comunicación, asuntos que constituyen la base de las orientaciones actuales en psicología social.

Si bien los trabajos en psicología ambiental no han logrado despojarse por completo de una herencia positivista, estos apuntan a lo que se ha planteado como la raíz de los problemas ambientales. El modelo de conexión con la naturaleza que propone Schultz (2002) es un ejemplo de que hay una psicología que no está lejos de la consideración de la cosmovisión occidental y de la separación ontológica hombre-naturaleza como ideologías responsables en la crisis ambiental. Schultz sostiene que la tecnología que caracteriza a las sociedades industrializadas ha contribuido a separar o a alienar al hombre con respecto a la naturaleza, interfiriendo en una conexión hipotética. La conexión con la naturaleza sería una experiencia esencialmente de carácter psicológica “Un análisis psicológico de la inclusión -dice Schultz- se centra en el entendimiento que tiene un individuo del lugar que ocupa en la naturaleza, así como el valor que le otorga a la naturaleza y sus acciones que tienen un impacto en ella”. El modelo de conexión con la naturaleza que propone este autor se compone de tres elementos, a saber: 1) Conexión (Conectedness) que se refiere al nivel en el que un individuo incluye a la naturaleza en las representaciones cognitivas de sí mismo; 2) Preocupación (Caring), una dimensión afectiva que incluye sentimientos de intimidad y de cercanía por la naturaleza y que está esencialmente referida a la medida en la que las personas se sienten responsables por la naturaleza; y 3) Compromiso (Commitment) que corresponde a una dimensión conductual que apunta a la motivación para actuar en el beneficio de la naturaleza. Estos tres componentes relacionados entre sí proporcionarían un marco para analizar la relación hombrenaturaleza a través de la medición estadística de cada una de las variables.

El gran mérito de este modelo es apuntar al problema central de la crisis ecológica. Se podría decir que “da en el clavo” en tanto señala que si la psicología quiere promover acciones sustentables debe estudiar e intervenir esta compenetración con la naturaleza. Sin embargo el modelo de Schultz, así como la mayoría de los abordajes de la psicología ambiental peca del mismo principio que critica. Al formular los constructos de conexión, preocupación y compromiso como componentes medibles de un fenómeno psicológico, esta teoría tiende a objetivar y a fragmentar una relación de por sí es integrada y compleja. La creación de un lenguaje para nombrar componentes específicos de este fenómeno implica la construcción de entidades susceptibles de ser concebidas como separadas del sujeto conocedor. A esta operación destinada a dividir el mundo entre sujeto y objeto a través del lenguaje especializado, Pablo Fernández (1994) la denomina epistemología de la distancia y la identifica con el cientificismo que ha encarnado la idea de progreso económico a ultranza basada en un el control y manipulación de los objetos de la naturaleza. 


\section{El papel de las narrativas}

Si el problema ambiental está definido en términos de una conciencia global, que trasciende a un problema individual y a las meras respuestas técnicas y científicas, para la comprensión de los fenómenos psico-sociales vinculados a lo que se ha denominado la crisis ecológica, se debe recurrir a una óptica que supere los abordajes teóricos y metodológicos centrados en la noción de individuo. Las teorías de la narrativa en psicología social constituyen una aproximación epistemológica pertinente que supera el antagonismo entre la realidad socio-cultural exterior y la realidad psicológica interior del sujeto, atendiendo al universo simbólico que las personas utilizan en la construcción y búsqueda de sentido de sí mismo y del mundo que los rodea.

Jerome Bruner (1991) plantea que la narración es una forma de pensamiento humano además de un fuerte componente de los procesos intersubjetivos. Esta consiste en la producción de relatos a través de los cuales las culturas producen significados que son compartidos y negociados por sus miembros, de modo de explicar los sucesos del mundo social y hacerlos comprensibles ante los otros y ante sí mismos. El recurso narrativo además de ordenar la experiencia individual por medio de la organización de episodios significativos, contribuye a cohesionar las creencias y valores compartidos, facilitando su transmisión en la cultura. A través de la narración, las personas pueden explicar y significar aquellos sucesos que resultan inhabituales, incomprensibles e inesperados dentro de un orden cultural, que Bruner llama sucesos no canónicos. Es decir que la narrativa permite hacer comprensible aquello que resulta ajeno al mundo cultural.

El estudio de las narrativas del sí mismo tiene importantes alcances en el conocimiento de las acciones sustentables. Desde esta aproximación los sujetos al relatar sus experiencias remiten los conceptos del dominio de la sustentabilidad un cierto orden sociocultural, que corresponde al de su propio ámbito cotidiano. El enfoque narrativo permite contextualizar las acciones a través de la creación de sentidos específicos. Esta perspectiva le otorga protagonismo a las versiones de las propias personas, permitiendo el despliegue subjetivo y validando relatos que la racionalidad oficial desestima tales como mitos, creencias, saberes culturales, etc., que por lo demás abundan en relación a la naturaleza. En este sentido puede decirse que la narración asigna al sujeto un papel de agente activo en la construcción de sus conocimientos, a la vez que descarta que la naturaleza y los conceptos afines tengan un significado absoluto.

Si la naturaleza es parte fundamental en la construcción del sí mismo, encontraremos que el mar, la montaña, los ríos, el ganado o los peces estarán presentes en la construcción de sentido de la experiencia pasada y presente, así como la proyección futura. Los elementos de la naturaleza pueden ocupar un lugar y un valor importante en cómo el sujeto relata y explica su experiencia, constituyendo verdaderas matrices narrativas a partir de las que se despliegan las historias y sentidos de vida de las personas. 
La posibilidad de instaurar una relación de semejanza con los objetos que componen nuestro mundo pasa por una capacidad de instituir significados que aquí hemos denominado construcción narrativa. ¿Qué lugar ocupo yo en la naturaleza?, ¿ Constituye realmente el medioambiente un fin en sí mismo?, ¿Es la sustentabilidad un criterio para fijar las pautas de consumo y para definir nuestras formas de vida?, son preguntas fundamentales que, en este sentido, se hacen visibles desde una aproximación narrativa. Una vez que se considera a la naturaleza y demás objetos del mundo como entidades similares al yo, la narración puede mediar un diálogo con este mundo colectivo. Operación esencialmente narrativa en tanto es una construcción de sentido y que Fernández (1994) llama la epistemología del encantamiento. "Una epistemología del encantamiento -dice este autor- inaugura un planeta con el que se puede dialogar y negociar, con el que hay que tener tacto y al que no se le puede herir impunemente, porque de la misma manera que nosotros estamos pensando y sintiendo al planeta, el planeta nos está pensando y sintiendo a nosotros” (Fernández, 1994: 275)

\section{Conclusión}

Generalmente las contribuciones de las ciencias sociales en torno al modelo de desarrollo sustentable se han desarrollado a partir de modelos basados en una lógica que aún proclama al individuo y a la objetividad como valores ideológicos. Como consecuencia de esto podemos plantear que se conserva la oposición ontológica entre sujeto -objeto y otras dualidades de la cosmovisión moderna.

Si tal como se ha planteado desde la ética ambiental en adelante, los problemas que afectan al planeta más que con recursos técnicos y científicos deben ser enfrentados con un cambio de conciencia global, entonces debemos atribuir al conocimiento científico la responsabilidad de buscar modelos epistemológicos alternativos que tiendan hacia la integración de la realidad y hacia el pensamiento colectivo. Las ciencias sociales deben aprovechar su rol de constructoras de discurso para realizar esta subversión epistemológica.

En psicología, la teoría de la narrativa del sí mismo es una alternativa promisoria para superar los esquematismos del paradigma positivista con que se han abordado tradicionalmente estos temas. Esta perspectiva reconoce la importancia de la cultura y de la subjetividad en la construcción de la realidad social y les asigna un carácter de agente social activo a las personas en la construcción del conocimiento. La construcción de narrativas permite el despliegue de subjetividades alternativas a la racionalidad dominante, así como la creación de un sentido de semejanza y solidaridad con los objetos del mundo. 


\section{Bibliografía}

Bruner, J. (1991), Actos de Significado. Más allá de la revolución cognitiva, Alianza, Madrid.

Brundtland, G. H. (1987), Our common future (Report for the World commission on Environment and Development, United Nations), Oxford University Press. Oxford.

Descola, P. \& Palssons, G. (eds.) (1996), Nature and Society: Anthropological perspectives, Routledge, London.

Fernández, Pablo (1994), La Psicología Colectiva: Un Fin de siglo más tarde, Anthropos, Michoacán.

Garling, T. y Golledge, R. (eds.) (1993), Behaviour and Enviroment: Psychological and Geographical Approaches, North Holland, Amsterdam.

Howard, G. (2000), “Adapting human lifestyles for the $21^{\text {st }}$ century”. American Psychologyst, 55(5), pp. 509-515.

Ibáñez, T. (1994), Psicología Social Construccionista, Universidad de Guadalajara, México.

Kaplan, S. y Kaplan, R. (1982), Cognition and Enviroment, Praeger , New York.

Lindón, A. (1999), “Narrativas autobiográficas, memoria y mitos: una aproximación a la acción social”. (pp. 295-310) Economía, Sociedad y Territorio vol. II $\mathrm{N}^{\circ} 6$.

Ruckelshaus, W. (1989), “Toward a Sustainable World” en Scientific American $\mathrm{N}^{\circ}$ 261, pp. 166-174.

Schultz, P. W. (2002), "Inclusión with nature: The Psychology of HumanNature Relations” en Schmuck, P. \& Schultz, W. P. (eds.) Psychology of sustainable development Kluwer, Boston.

Vlek, C. \& Steg, L. (2007), "Human Behaviour and Environmental Sustainability: Problems, Driving Forces, and Research Topics” en Journal of Social Issues, Vol.63, No. 1.

Weisenfeld, E. (2003), “La Psicología Ambiental y el desarrollo sostenible. Cuál psicología ambiental? Cuál desarrollo sostenible?” en Estudos de Psicología 2003, 8 (2), pp. 253-261.

White, L. (1967), “The Historical Roots of our ecological crisis” en Science 155, pp. 1203-1207. 